\title{
6-day-old term infant with fever and lethargy
}

\author{
Gangaram Akangire ${ }^{1,2^{*}}$, Archana Kulkarni ${ }^{3}$, Winston Manimtim¹, 2
}

\author{
${ }^{*}$ Correspondence \\ Gangaram Akangire, MD, MS, FAAP \\ Section of Neonatology Children's \\ Mercy Hospital-Kansas City 2401 \\ Gillham Road Kansas City, MO 64108 \\ Email: gakangire@cmh.edu \\ Phone: 816-234-3591 \\ Fax: 816-302-9887 \\ 1 Division of Neonatology Children's \\ Mercy Kansas City, Kansas City, MO \\ 2 University of Missouri-Kansas City \\ ${ }_{3}^{3}$ Samuel Rodgers Health center, Kansas \\ City \\ Received: Mar 16, 2018 \\ Accepted: Apr 25, 2018 \\ Published: Apr 28, 2018
}

\begin{abstract}
Osteomyelitis in the neonatal period can be a diagnostic challenge, especially if it is mimicking brachial plexus palsy. We present a unique case of 6-day-old term infant with lethargy and fever diagnosed with Group B Streptococci (GBS) sepsis and meningitis. Few days later, infant developed right arm immobility leading to hematogenous spread of GBS to right humerus, leading to osteomyelitis and septic arthritis. Infant was treated with intravenous Ampicillin for 4 weeks with full arm mobility and full recovery from sepsis and meningitis. This case provides several clinical vignettes that may be crucial in timely diagnosis and treatment of osteomyelitis and septic arthritis that may be useful in avoiding long-term complications.
\end{abstract}

Keywords: Neonatal osteomyelitis, Neonatal sepsis, Brachial plexus palsy, Neonatal fever.

\section{Case Presentation}

A term baby boy was born in an outlying hospital to a gravida 1 para 1 mother by spontaneous vaginal delivery. The mother had uncomplicated prenatal course, and her prenatal laboratory findings including group B streptococci (GBS) were unremarkable. The infant remained in the well-baby nursery with the mother and was discharged appropriately at 48 hours of life. The infant presented to emergency room on day of life (DOL) 6 with history of fever with maximum temperature of $102^{\circ} \mathrm{F}\left(38.9^{\circ} \mathrm{C}\right)$ and lethargy with decreased oral intake for 1-2 days. He was evaluated in the emergency room and was admitted to the pediatric floor with the diagnosis of suspected sepsis, and empiric ampicillin at $200 \mathrm{mg} / \mathrm{kg} /$ day divided every 12 hours and cefotaxime at $200 \mathrm{mg} / \mathrm{kg} /$ day divided every 6 hours were started appropriately. Fifteen hours after admission, blood culture showed growth of group B streptococci (GBS) sensitive to ampicillin. Lumbar puncture was unsuccessful, so the infant was transferred to a tertiary care NICU for further management. Lumbar puncture later showed significantly elevated white blood cell (WBC) count and was consistent with bacterial meningitis. The infant had stable vitals and was feeding well. He was intermittently irritable, but his physical examination was unremarkable. The infant was in the NICU for 21 days of IV antibiotics administration with ampicillin and cefotaxime for 48 hours and after growth of GBS, cefotaxime was discontinued and ampicillin was completed for 21 days. Initial complete blood cell count (CBC) showed WBC of $25 \times 10^{3}$ cells $/ L$ and Creactive protein (CRP) was $40 \mathrm{mg} / \mathrm{dl}$. On DOL 11, the bedside nurse notes that the infant had pain in his right arm upon movement with decreased motion. $X$ ray of the right clavicle and humerus was done and was non-contributory. It was presumed that the infant had brachial plexus injury and physical therapy was initiated for arm motion. On DOL 17, he was extremely irritable and was unable to move his arm (Fig 1). Magnetic resonance imaging (MRI) of the right arm revealed the diagnosis. 


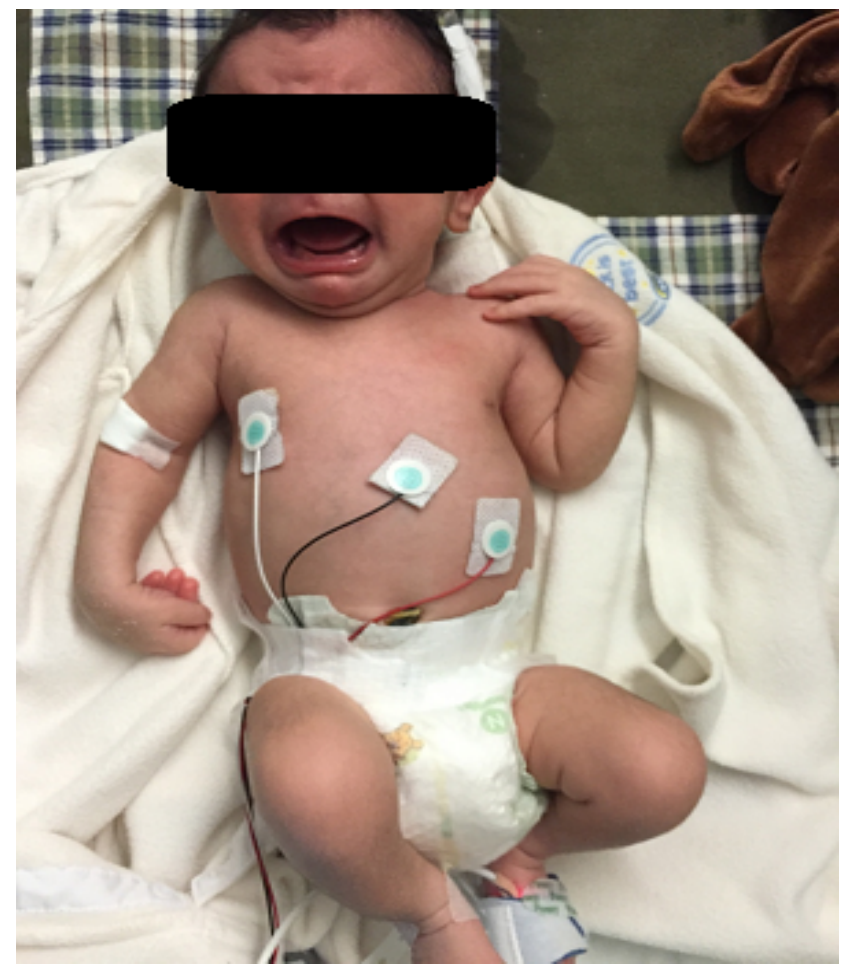

Fig 1: Infant with right arm weakness mimicking brachial plexus injury.

\section{Diagnosis}

MRI of the right arm revealed osteomyelitis of the right humeral head and fluid in the right shoulder joint, confirming the diagnosis of osteomyelitis and septic arthritis (Fig 2).

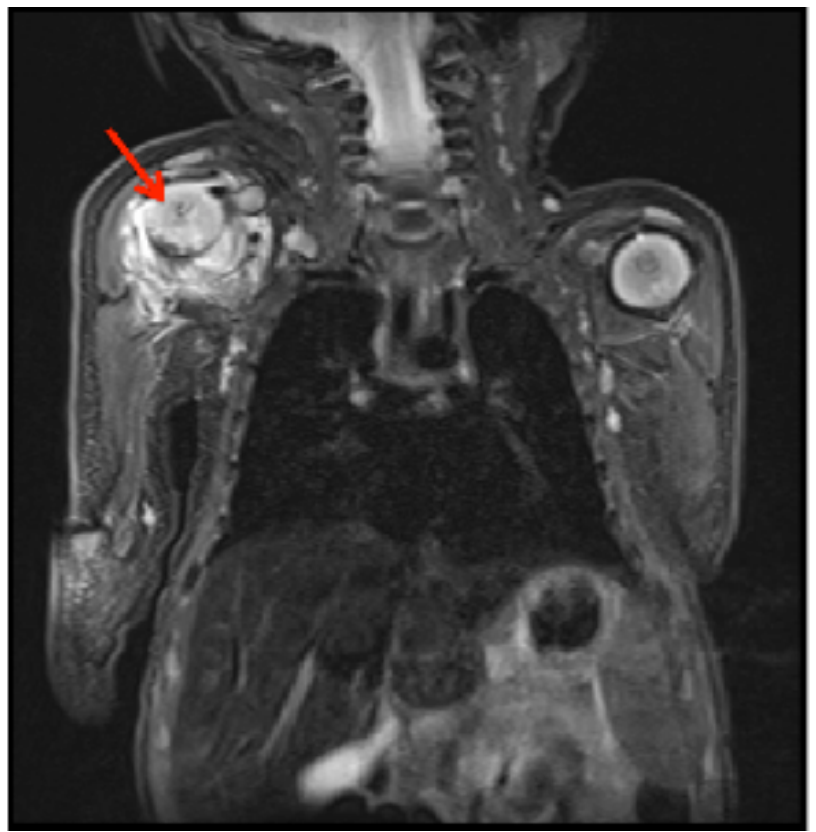

Fig 2: Magnetic resonance imaging showing right humeral osteomyelitis and septic arthritis. Red arrow indicates affected joint.

\section{Discussion}

Neonatal osteomyelitis is fairly uncommon, with the reported incidence of 1 in 5000 to 1 in 15000 infants depending on the geographic location [1]. Bacteremia due to variety of causes is the most important factor leading to neonatal osteomyelitis. Premature infants have several other risk factors that lead to bacteremia, such as umbilical vessel catheterization, venous cut down, periumbilical skin infections, heel sticks, and urinary tract infections. Most bone infections in neonates are localized to the metaphysis of long bones. This localization is due to the sharp turn taken by the arterioles in the metaphysis, which causes slowing of blood circulation that leads to seeding of circulating bacteria in the metaphysis [2].

The femur and tibia are the most commonly affected bones in neonatal osteomyelitis, followed by the humerus and fibula [3] . The hip, elbow, knee, shoulder, ankle, and phalanx are the joints affected in neonates [1]. Young infants have a higher propensity to develop maxillary bone infections compared to older children. Although the exact pathogenesis is unknown, maxillary bone infections usually start with conjunctivitis, leading to periorbital abscess and eventually involve maxillary bone [3] . The most common bacteria leading to neonatal osteomyelitis and septic arthritis are methicillinsensitive staphylococcus aureus (MSSA), methicillinresistant staphylococcus aureus (MRSA), and group B streptococcus (GBS). Less common bacterial causes are Escherichia coli, Klebsiella pneumonia, Enterobacter cloacae, Salmonella enteritidis, and Citrobacter freundii [3]. GBS has a high propensity to affect the humerus and shoulder joint, as in our case. Studies from 1950 to 1970 indicate that staphylococcus was the most common cause leading to neonatal osteomyelitis, but GBS emerged to be the leading cause from 1970 to 1990 [4]. After 1990, due to intrapartum GBS prophylaxis, MSSA and MRSA became the leading causes again.

Most infants have non-specific clinical symptoms such as irritability, lethargy, poor feeding, and temperature instability. Specific symptoms may take some time to occur and can include limited movement and discomfort with movement (present in our case), and pseudoparalysis (present in our case). Localized tenderness, erythema, swelling, and 
warmth at the affected site are fairly late manifestations (not present in our case) [2].

To diagnose neonatal osteomyelitis and septic arthritis, a detailed maternal and neonatal history is important, along with physical examination and high index of suspicion. History of difficult delivery/ instrumental delivery is important if the presentation includes decreased motion or pain of extremity. In the setting of a negative history of difficulty/ instrumental delivery and the presence of bacteremia with decreased motion of extremity or pain with motion, osteomyelitis should be high on the list of differential diagnoses. Physical examination can be helpful if localized swelling, erythema, or warmth is present, but these signs may not be present in the early period.

Laboratory studies such as CBC, CRP, and erythrocyte sedimentation rate (ESR) may be helpful in early diagnosis. No specific neonatal studies are done on inflammatory markers, but pediatric studies show that CRP increases rapidly on day 1 within hours after infection and ESR increases on day 1-2 after infection. CRP alone has a sensitivity of 95\%, ESR alone has a sensitivity of $94 \%$, and combined CRP and ESR has a sensitivity of up to $98 \%$ to diagnose bone infections in previous studies [5]. CBC can also be used as an adjunct in diagnosis of bone infections. Once osteomyelitis or septic arthritis is suspected, MRI is warranted to confirm the diagnosis and is the gold standard test for bone infections [3] . Bone scan has some value in older children, but has a high false negative rate in neonates. Computed tomography scan is valuable in diagnosing bony deformities and can pick up bone destruction and periosteal new bone formation, but has less value in early osteomyelitis when soft tissue details are required. Ultrasonography may be useful in diagnosis of septic arthritis and may help in guiding the needle for drainage. If MRI is positive for bone infection, an orthopedic consult should be pursued as soon as possible for further management.

Nafcillin or oxacillin and gentamicin, or vancomycin (if MRSA is suspected) and gentamicin can be the empiric antibiotic regimen. Ceftazidime or cefotaxime can be added if enteric bacteria are suspected [3] . Joint decompression should be performed as soon as possible in septic arthritis to prevent further irreversible damage to the growth plate [3] . Damage to the growth plate causes growth disparities and is usually seen around 9-10 years of age. Infection of the hip joint causes avascular necrosis of the femoral head and can also lead to degenerative arthritis in the affected joint. Outcomes are usually better with rapid decompression and early antibiotics. Antibiotics are usually given for 4-6 weeks.

\section{Patient Course}

On DOL 18, the infant was taken to the operating room by orthopedic surgeons and aspiration of minimal bloody fluid was done from the joint with minimal pus. Microscopic examination of aspirated fluid revealed increased WBC count, but no growth of organisms, possibly because the infant had been on antibiotics for several days. In accordance with infectious disease specialist recommendations, the infant was treated with intravenous ampicillin at $200 \mathrm{mg} / \mathrm{kg} /$ day divided every 12 hours for 4 weeks and was discharged home with full right arm motion and normal findings on physical examination. Ampicillin was the drug of choice in our patient since GBS was isolated in blood culture and the humeral location of osteomyelitis was thought to be consistent with GBS osteomyelitis []]. Ampicillin penetrates the bone at a level of $\sim 5-20 \%$ of serum and the bone levels are high enough to target minimum inhibitory concentration (MIC) of bacteria. Antibiotic penetration is better in inflamed bones compared to normal bones [ $\underline{6}]$.

\section{Summary}

A. Neonatal osteomyelitis and septic arthritis are relatively uncommon infections.

B. Bacteremia is a significant risk factor for neonatal osteomyelitis and septic arthritis.

C. Thorough maternal and neonatal history (focus on difficult/instrumental delivery), detailed physical examination, and high index of suspicion are important in early diagnosis and treatment.

D. Elevated CRP and ESR together have up to $98 \%$ sensitivity for diagnosis.

E. MRI is the gold standard imaging modality for early diagnosis.

F. Empiric antibiotics in the neonatal period should cover MSSA, gram-negative organisms and GBS. 
G. Neonatal osteomyelitis and septic arthritis can lead to irreversible damage if not diagnosed and treated promptly.

\section{Acknowledgement}

Authors thank Medical Writing Center at Children's Mercy for their help in editing the manuscript.

\section{Copyrights}

(c) Gangaram Akangire et al, 2018; licensee OA Journal of Case Reports. This is an Open Access article distributed under the terms of the Creative Commons Attribution License (http:// creativecommons.org/licenses/by/4.0), which permits unrestricted use, distribution, and reproduction in any medium, provided the original work is properly credited.

\section{References}

1. Narang A, Mukhopadhyay K, Kumar P, et al. (1998) Bone and Joint Infection in Neonates. Indian J Pediatr 65: 461-464.

2. McPherson DM (2002) Osteomyelitis in the Neonate. Neonatal Netw 1: 9-22

3. Fisher R (2011) Neonatal Osteomyelitis. NeoReviews 12: 374-380.

4. Edwards MS, Baker CJ, Wagner ML, et al. (1978) An Etiologic Shift in Infantile Osteomyelitis: The Emergence of the Group B Streptococcus. J Pediatr 93: 578-583.

5. Paakkonen M, Kallio M, Kallio PE, et al. (2010) Sensitivity of Erythrocyte Sedimentation Rate and C-reactive Protein in Childhood Bone and Joint Infections. Clin Orthop Relat Res 468: 861-866.

6. Spellberg B, Lipsky B. (2012) Systemic Antibiotic Therapy for Chronic Osteomyelitis in Adults. Clinical Practice 54: 393-407. 